\section{Nanowires by Step Decoration}

\section{F.J. Himpsel, T. Jung, A. Kirakosian, J.-L. Lin, D.Y. Petrovykh, H. Rauscher, and J. Viernow}

\section{Self-Assembly of One- Dimensional Nanostructures}

Recent advances in the control of thin films and surfaces have brought an intriguing question within reach: Is it possible to tailor the electronic properties of solids by controlling them layer by layer or row by row? Customized molecules are commonplace in biochemistry. Can the same idea be brought to bear on solids and electronic materials? Electronic properties of semiconductor devices have been controlled by heterostructures, quantum wells, and superlattices. Magnetism as a cooperative phenomenon lends itself to manipulation in small structures, where neighbor atoms can be replaced systematically by species with stronger or weaker magnetism. ${ }^{1,2}$ In fact, a class of magnetic/nonmagnetic multilayers termed spin valves has recently been introduced into commercial read heads for magnetically stored data. The optimum thickness of their active region lies in the single-digit-nanometer regime.

The smallest nanostructures may be viewed as objects consisting only of interfaces with no bulk behind them. More typically, single-digit-nanometer dimensions are sufficient for realizing the benefits of structuring (e.g., operating a quantum-well device at room temperature). This regime is difficult to reach with lithography methods, particularly when macroscopic a mounts are to be fabricated. Self-assembly becomes the method of choice.

This article focuses on the self-assembly of linear nanostructures such as arrays of stripes and strings of dots. There are a variety of self-assembly methods to choose from. Elongated structures of ten grow spontaneously at anisotropic surfaces. Adsorbates can form atomic rows by diffusing along atom rows on the facecentered cubic (fcc) (110) surface. ${ }^{3}$ Such elongated features grow on semiconductor surfaces as well, for example, in homoepitaxy of $\mathrm{Si}$ on the anisotropic
Si $(100)(2 \times 1)$ surface, or by adsorption of alkali and alkaline earth atoms. Wires perpendicular to a surface have been obtained by electroplating metals into channels across a thin film (e.g., etched nuclear tracks in a polymer ${ }^{4}$ and anodized pores in alumina ${ }^{5}$ ). Whiskers can be obtained by seeding them at the surface. ${ }^{6}$

Here, we focus on concepts that utilize a stepped surface as a template (Figure $1^{7,8}$ ). Adsorbed atoms or molecules have a tendency to stick to the step edges, where they find extra bonding partners for lateral bonds. Ideally, one hopes to achieve a step-flow growth mode, ${ }^{9}$ where stripes grow along the step edges in a row-by-row fashion (Figure 1a). Such a method is attractive because it allows for two controllable process parameters: (1) the coverage determines the stripe width, as in many of the other self-assembly methods; and (2) the miscut angle of the substrate determines the spacing between the stripes. Simple step-flow growth can be extended into a more "universal" method ${ }^{8}$ that emulates silicon microlithography on the nanoscale (Figure 1b). Initially, an inert mask is produced by step-flow growth and acts as the equivalent of the photo- resist. Selective deposition (or etching) in the areas not protected by the mask produces features with controllable vertical aspect ratios. Silicon-based techniques are able to take advantage of a vast array of well-tested processing methods.

In the following sections, we will explore how such an idealized view holds up at real surfaces. We will encounter a wide variety of growth modes when studying the self-assembly of onedimensional objects in a two-dimensional world. Not only wires, but also linear arrays of dots can be fabricated with dimensions in the single-digit-nanometer regime and areal densities of a few teradots per square inch. Silicon substrates will be considered in detail. In all of these studies, new chemical-imaging methods are used in combination with scanning tunneling microscopy (STM) for observing the redistribution of adsorbate and substrate atoms on the nanometer scale.

\section{Growth Modes at Stepped Surfaces}

The idea of obtaining nanowires by step-flow growth was tested early on for the GaAs/GaAlAs system. ${ }^{9}$ Step-flow growth proceeds rather well for these materials because they are structurally and chemically matched. This method can be extended to more heterogeneous adsorbate/substrate combinations, as demonstrated for combinations of metals. ${ }^{7,810-13}$ It requires a substrate with high surface energy ( $W, M o$ ) and an adsorbate with low surface energy $(\mathrm{Cu})$ that does not alloy with the substrate. Figure 2 shows $3-\mathrm{nm}$-wide $\mathrm{Cu}$ wires on a stepped $M o(110)$ surface as an example.

Step-flow growth is not the only option. A surprising variety of additional growth modes can be identified and classified, as shown in Figure 3. All of the familiar equilibrium growth modes

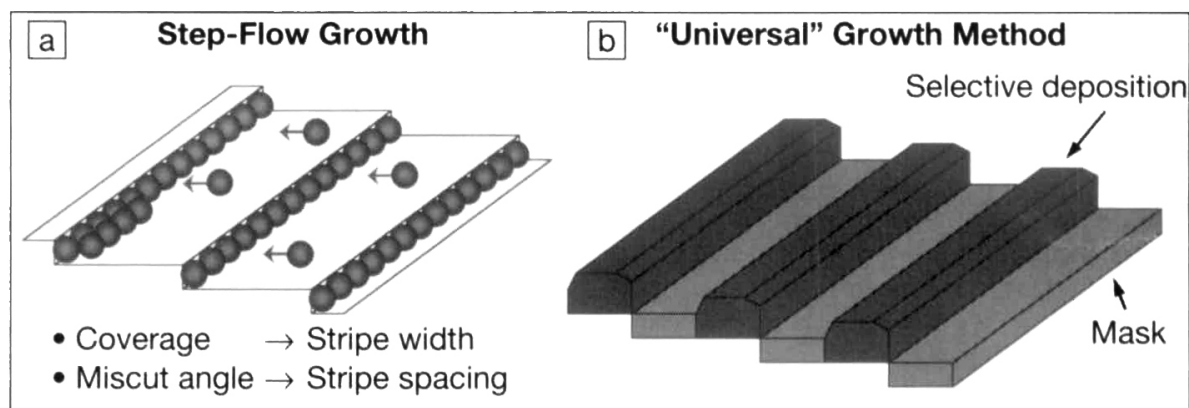

Figure 1. Concepts for fabricating nanowire arrays by self-assembly at stepped surfaces. (a) Stripes formed row by row by means of step-flow growth.

(b) "Universal" growth method involving a mask and selective deposition (or etching) in areas that are not protected by the mask. 


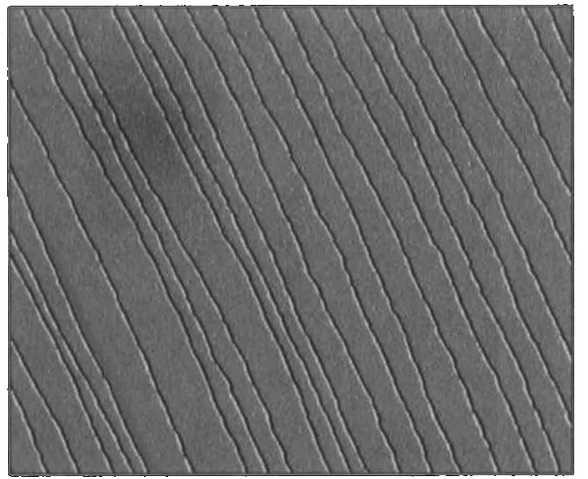

Figure 2. Copper wires ( $3 \mathrm{~nm}$ wide, $50 \mathrm{~nm}$ apart) grown by step flow on a stepped Mo(110) surface. In this scanning-tunneling-microscopy (STM) image, the derivative of the topography is shown. Copper appears bright as a result of resonant tunneling into a Cu surface state (thin white lines). The black lines are shadows of the steps.
All three equilibrium growth modes have been found at stepped metal sur faces. Figure $4 \mathrm{a}$ shows $\mathrm{Cu}$ growing row by row on stepped $M o(110)$. It grows in the Stranski-Krastanov mode on W(110), as shown in Figure $\mathbf{4 b}$. The first row of $\mathrm{Cu}$ atoms decorates the step edge (as shown in Reference 11), whereas additional $\mathrm{Cu}$ grows in monolayer-height islands at the step edges. Co and Fe grow in the island mode on $\mathrm{Cu}$, forming strings of islands that are more than a layer high. ${ }^{14}$ The island growth of reactive $\mathrm{Co}$ on a noble metal is not surprising: $\mathrm{Co}$ balls up into islands to keep as much as possible of the surface exposed to $\mathrm{Cu}$, which has lower surface energy. Such a growth mode can be expected, in general, when attempting to grow highenergy ferromagnets on low-energy substrates such as noble metals and insulators. The balance between row-by-row and Stranski-Krastanov growth is more delicate. In both cases, one has a material

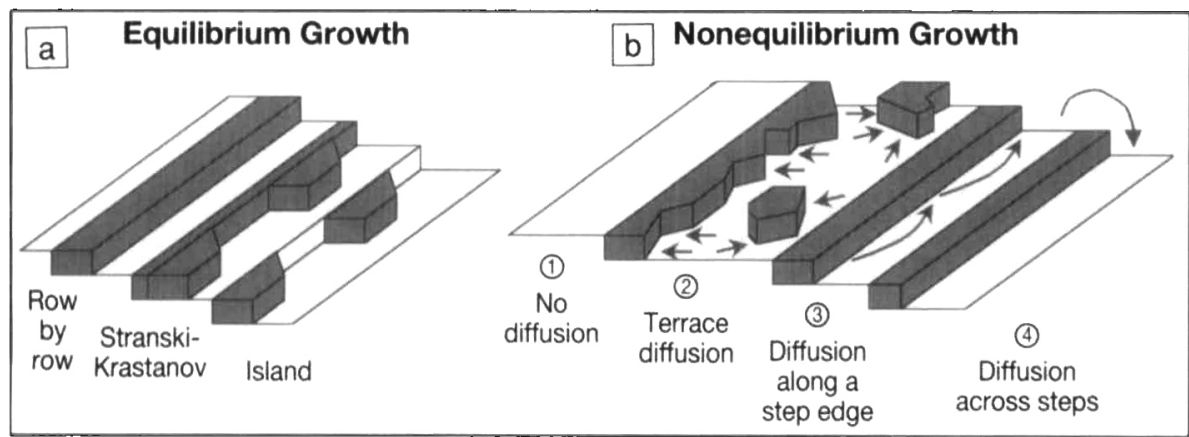

Figure 3. Growth modes observed at stepped surfaces. In addition to the usual equilibrium growth modes (a), one observes at least four nonequilibrium growth modes (b) for Cu on stepped Mo(110). They are characterized by thermal activation of diffusion on the terraces, along the step edges, and across the steps. reappear in a two-dimensional world, such as row by row (the equivalent of layer by layer), Stranski-Krastanov (layer and island growth), and island growth (Figure 3a). In addition, four nonequilibrium growth regimes can be identified at lower temperatures and higher growth rates. They are characterized by the suppression of various diffusion processes at reduced temperatures, such as diffusion across terraces, diffusion along step edges, and diffusion across steps (Figure 3b). The growth mode is rather sensitive to the combination of adsorbate and substrate, as demonstrated in Figure 4. $\mathrm{Cu}$ forms stripes on $\mathrm{Mo}(110)$ and strings of two-dimensional islands on W(110). with low surface energy $(\mathrm{Cu})$ adsorbed on a substrate with high surface energy (Mo, W). On W(110), the misfit strain with the substrate causes the $\mathrm{Cu}$ stripes to break up at misfit dislocations. On $\mathrm{Mo}(110)$, the $\mathrm{Cu}$ stripes remain continuous in order to minimize their step energy. One might speculate that the misfit strain wins on $W(110)$ because of the stronger $\mathrm{W}-\mathrm{Cu}$ bond. The $\mathrm{Cu}-\mathrm{Mo}$ bond is weaker and shifts bond strength toward the $\mathrm{Cu}-\mathrm{Cu}$ bond, which determines the $\mathrm{Cu}$ step energy.

The nonequilibrium growth modes show up very nicely for $\mathrm{Cu}$ on stepped Mo(110) at lower temperatures. The schematic view in Figure 3 can be quantified by statistical analysis of the growth pat- terns, as shown in Figure 5. With increasing growth temperature, successive diffusion processes become activated and give rise to four nonequilibrium growth regimes: ${ }^{12}$

- Regime 1: At the lowest growth temperatures, small islands are formed that sweep up adsorbed $\mathrm{Cu}$ atoms within a diffusion length around the nuclei (not shown).

- Regime 2: When the diffusion length becomes larger than a terrace width, $\mathrm{Cu}$ stripes are formed along the step edges. Initially, the stripe-width distribution is Poisson-like when measured in units of atomic rows (Figure 5b, top). $\mathrm{Cu}$ atoms become attached to a stripe wherever they land. Another characteristic of Regime 2 (and of Regime 3 ) is a stripe width proportional to the width of the underlying terrace (Figures $5 a$ and $\mathbf{4 a}$,
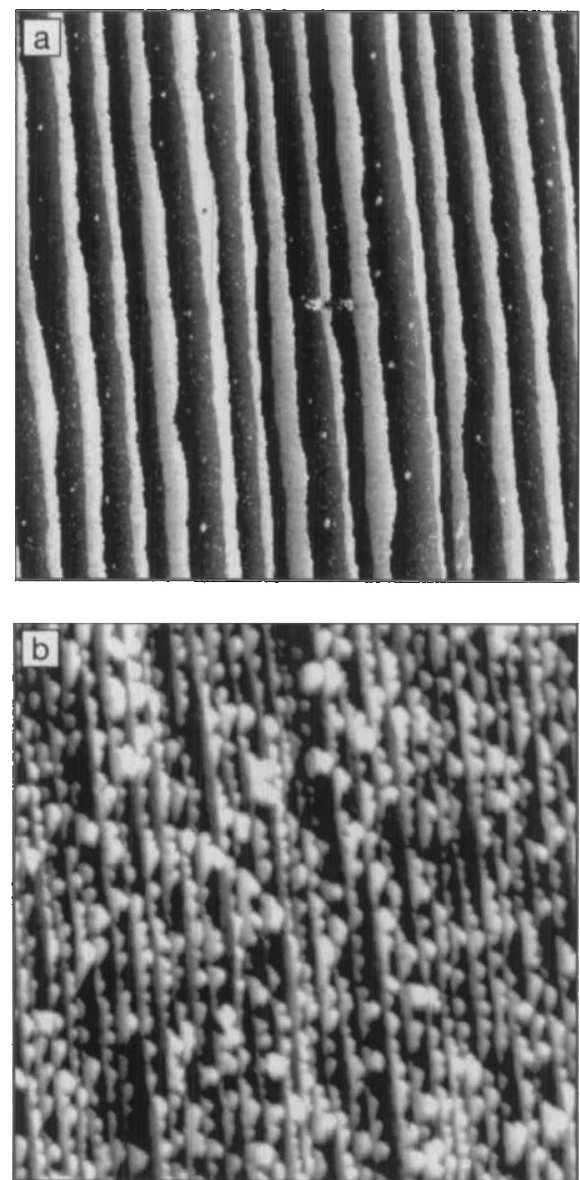

Figure 4. Different growth modes of Cu on (a) stepped Mo( 110 ) and (b) stepped W(110). In STM images $\left(700 \times 700 \mathrm{~nm}^{2}\right)$, Cu appears bright as a result of tunneling into a $\mathrm{Cu}$ surface state. From References 7 and 8. 

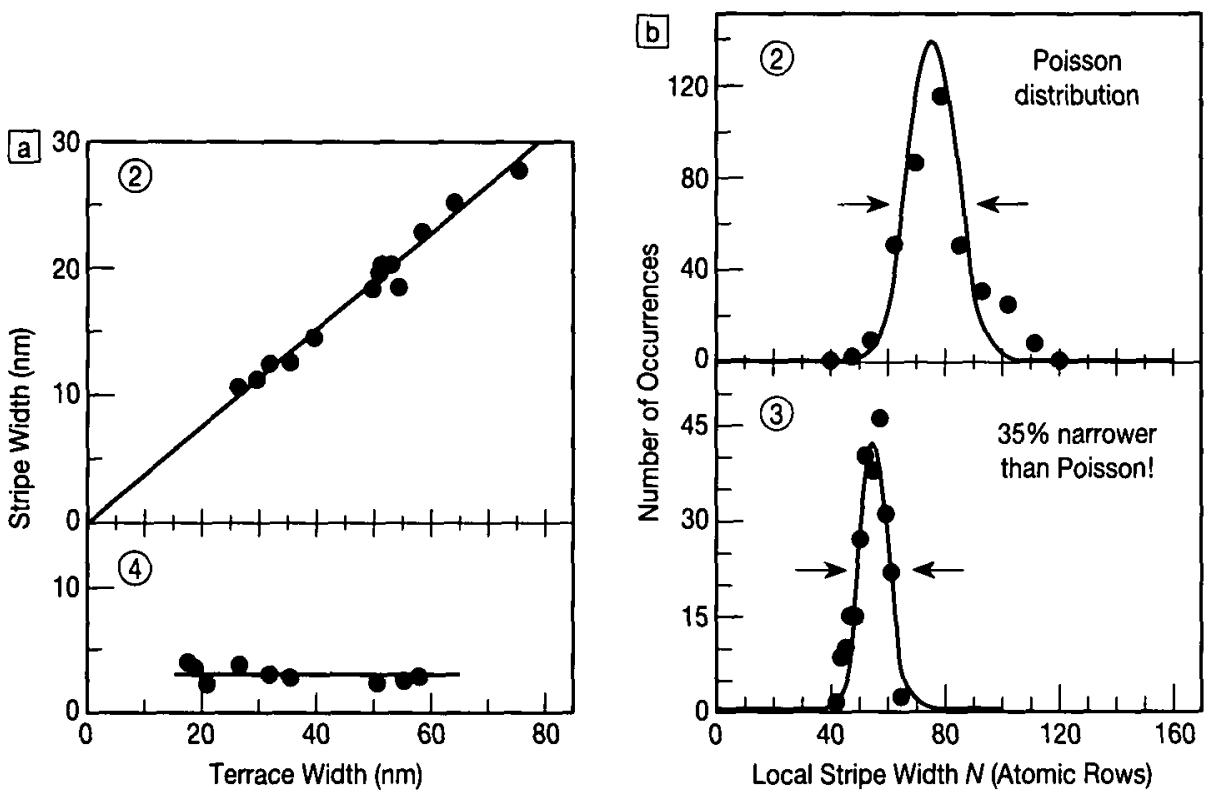

Figure 5. Statistical analysis of nonequilibrium growth modes for Cu on stepped Mo(110). Circled numbers denote the modes identified in Figure 3.

(a) Stripe width versus terrace width. In Mode 2, the Cu stripe width is proportional to the Mo terrace width, since all Cu atoms landing on a terrace are swept off into the same stripe. In Mode 4, the stripe width is independent of the terrace width, since Cu atoms are able to cross step edges and find the optimum sites closest to the Mo step edges.

(b) Wire width distributions. In Mode 2, the width distribution along a stripe corresponds to a Poisson distribution when measured in atomic rows, indicating that $\mathrm{Cu}$ atoms remain wherever they land along a Cu stripe. The arrows designate $a$ standard deviation $\pm \sigma / N\rangle= \pm 14 \%$. In Mode 3, the distribution is narrower than the stochastic Poisson distribution, indicating that diffusion along the Cu step edges smoothens the stripes $( \pm \sigma /\langle\mathrm{N}\rangle= \pm 8 \%)$. From Reference 12 .

top). All of the $\mathrm{Cu}$ atoms landing on a particular terrace diffuse toward the same stripe. Large terraces support wide stripes.

- Regime 3: After activating the diffusion of $\mathrm{Cu}$ along the stripe edge, the steps are straightened out, and the width distribution becomes narrower (35\% narrower than the stochastic Poisson width in Figure 5b, bottom). A reduced step length lowers the step energy.

- Regime 4: At the highest temperatures, diffusion across step edges becomes activated. The $\mathrm{Cu}$ atoms are able to redistribute themselves into the energetically most favorable sites, which apparently are close to the Mo step edges. The reason for this may be an inhomogeneous strain field resulting from lattice mismatch and strain relaxation at step edges. ${ }^{12}$ In this case, the stripe width becomes independent of the terrace width (Figure 2).

For molecular adsorbates, such as $\mathrm{CaF}_{2}$ on $\mathrm{Si}(111)$, an even richer set of growth modes evolves that depends not only on temperature (or supersaturation), but also on coverage. Stripes can attach themselves at the top of a step edge, instead of at the bottom, or can break up into regular arrays of dots (see later section on "Strings of Dots").

For quantitative measurement of width distributions, it is necessary to have an imaging technique that provides clear chemical contrast at the nanometer scale. We have developed chemically sensitive STM techniques that utilize resonant tunneling into metal-specific surface states. $^{13,15}$ Electrons at the Fermi level of the tip tunnel directly into an unoccupied surface state of the sample at specific bias voltages. In the case of $\mathrm{Cu}, \mathrm{W}$, and Mo, there are two types of elementspecific surface states: an image state whose energy is related to the work function, and a surface state near the Fermi level that produces high-resolution images at low bias voltage. For insulators, such as the calcium fluoride films dis- cussed in the next section, tunneling into the states at the conduction-band minimum provides chemical identification. ${ }^{16}$

\section{Universal Fabrication Methods}

With step-flow growth being limited to a few combinations of substrates and overlayers, it becomes desirable to develop more universal self-assembly methods. These methods should allow arbitrary substrates, overlayer materials, and stripe thicknesses. In particular, it would be very useful to have silicon as a substrate, not only to take advantage of the many silicon processing techniques that already exist, but also for future integration of nanodevices into silicon microchips. A general pathway to such a universal growth method is outlined in Figure $1 \mathrm{~b}$ It borrows the idea of an inert mask from $\mathrm{Si}$ microlithography and arrives at the following three-step process:

1. A stepped silicon surface forms the template.

2. An inert mask is deposited by stepflow growth.

3. Selective deposition or etching in the areas not protected by the mask creates the desired stripe array.

Next, we will give a status report for each of these steps.

Producing regular step arrays has been quite successful on the $\operatorname{Si}(111)(7 \times 7)$ surface. ${ }^{17,18}$ As demonstrated by the STM images in Figure 6, steps can be made extremely straight on $\mathrm{Si}(111)(7 \times 7)$, with a kink density as low as one in 20,000 edge atoms. ${ }^{17}$ This results from the large $\mathrm{ki}$ netic barrier for creating a kink, ${ }^{19}$ which requires adding seven atomic rows, two layers deep. The $\operatorname{Si}(111)(7 \times 7)$ surface consists of triangles with opposite stacking (staggered and eclipsed), giving rise to natural grooves at their boundaries (fine vertical lines, Figure 6). These coincide with the stable step edges. In order to obtain such straight edges, one has to produce a single $(7 \times 7)$ domain (out of 49 possible). As shown in References 17 and 18 , this can be achieved over large distances (hundreds of nanometers) by a specific annealing sequence. First, a clean Si surface is produced by heating above $1250^{\circ} \mathrm{C}$, where residual carbon diffuses into the bulk. A subsequent quench past the $(1 \times 1)$-to- $(7 \times 7)$ phase transition avoids step-bunching instabilities, and a long anneal at $850^{\circ} \mathrm{C}$ plus a slow cooldown creates large $(7 \times 7)$ domains and straight step edges. Another important factor for kink-free step arrays is the correct azimuthal orientation of the miscut. The tilt of the surface normal is $1^{\circ}$ from [111] toward [ $\overline{1} \overline{1} 2]$ for the samples used in Figures 7-9. The step spacing varies 


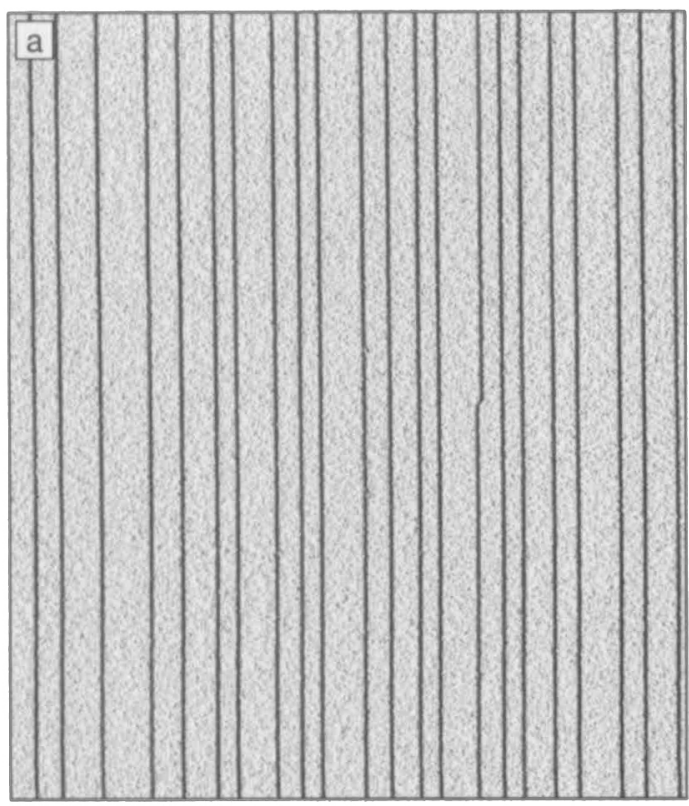

$17 \overline{\mathrm{nm}}$

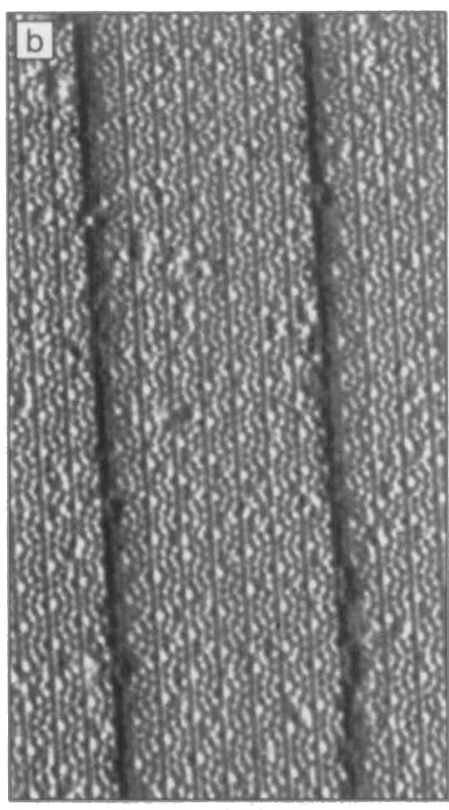

$17 \mathrm{~nm}$

Figure 6. Preparation of very straight steps on Si(111) $(7 \times 7)$. (a) The overview shows only a single kink in 20,000 edge atoms $\left(340 \times 390 \mathrm{~nm}^{2}\right)$. (b) The close-up $\left(40 \times 70 \mathrm{~nm}^{2}\right)$ shows atomically straight step edges following the natural grooves in the $(7 \times 7)$ surface that separate areas of opposite stacking. The derivative of the STM topography is shown, giving the appearance of a surface being illuminated from the left. After References 17 and 18.

somewhat for these $\mathrm{Si}(111)$ surfaces because of a weak step-step interaction. It is quantized in units of $2.3 \mathrm{~nm}$, which corresponds to the groove spacing of seven atomic rows. If one is interested in decoupled stripes, the variations in the spacing are irrelevant (as long as the steps are far enough apart). Having smooth edges is the key to creating smooth wires, and in that respect, the $\mathrm{Si}(111)(7 \times 7)$ surface is much more perfect than a metal surface (see Figure 2).

In the second step, stripes of epitaxial $\mathrm{CaF}_{2}$ and $\mathrm{CaF}_{1}$ have been produced ${ }^{20,21}$ by step decoration of $\mathrm{Si}(111)(7 \times 7)$. Such a structure is shown in Figure 7, where 7-nm-wide $\mathrm{CaF}_{2}$ stripes are arranged with a $15-\mathrm{nm}$ periodicity. The best $\mathrm{CaF}_{2}$ stripes are obtained after depositing between one and two monolayers of $\mathrm{CaF}_{2}$ with a brief postanneal above $800^{\circ} \mathrm{C}$. In this case, a complete $\mathrm{CaF}_{1}$ layer coats the $\mathrm{Si}(111)$ surface, and $\mathrm{CaF}_{2}$ stripes form on top of that layer, starting at the upper edge of the steps. ${ }^{20}$ The second valence electron of the $\mathrm{Ca}$ atoms in the $\mathrm{CaF}_{1}$ layer bonds to the broken surface bond of $\mathrm{Si}(111)$. The $\mathrm{CaF}_{2}$ stripes are all continuous and completely separated from each other. They avoid each other even after decreasing their spacing at a coverage of nearly two monolayers (Figure 8 ), which suggests that a repulsive interaction keeps the stripes apart. The counterintuitive attachment of deposited material at the top of a step edge, as well as the stripe-stripe repulsion, can be explained by a reversal of the stacking at the $\mathrm{CaF}_{1} / \mathrm{Si}(111)$ interface. ${ }^{20}$ The bond topology prevents $\mathrm{Ca}$ atoms from bonding to the uphill terrace.

The third step-that is, selective deposition or etching between the $\mathrm{CaF}_{2}$ stripes -is currently being explored. For selective deposition, one might consider chemical vapor deposition (CVD), electroplating, evaporation with subsequent diffusion across the inert $\mathrm{CaF}_{2}$, or shadowed evaporation. Shadowed evaporation might become useful with bunched steps that can be fabricated on $\operatorname{Si}(111)(7 \times 7)$ with a different miscut direction. ${ }^{18}$ These bunches are typically 1-2 $\mathrm{nm}$ high and $50 \mathrm{~nm}$ apart, giving rise to more robust structures that are still significantly below the reach of today's manufacturing technology. Progress has been made with selective CVD, where organic precursor molecules for CVD of metals have been deposited selectively in the valleys between the $\mathrm{CaF}_{2}$ stripes, ${ }^{22}$ as shown in Figure 8. At room temperature, the molecules adsorb everywhere (not shown), but at $300^{\circ} \mathrm{C}$, they remain only in the valleys, which consist of $\mathrm{CaF}_{1}$. This selectivity is explained by the smaller bandgap of $\mathrm{CaF}_{1}$ compared to $\mathrm{CaF}_{2}(2.4$ versus $12 \mathrm{eV})$, which gives a better match to the highest occupied and lowest unoccupied orbitals of the adsorbed molecules (typically $3 \mathrm{eV}$ ). The metallocenes are valuable precursors for depositing ferromagnetic materials $(\mathrm{Fe}, \mathrm{Co}, \mathrm{Ni})$, because they can be decomposed with ultraviolet light without incorporating carbon or oxygen.

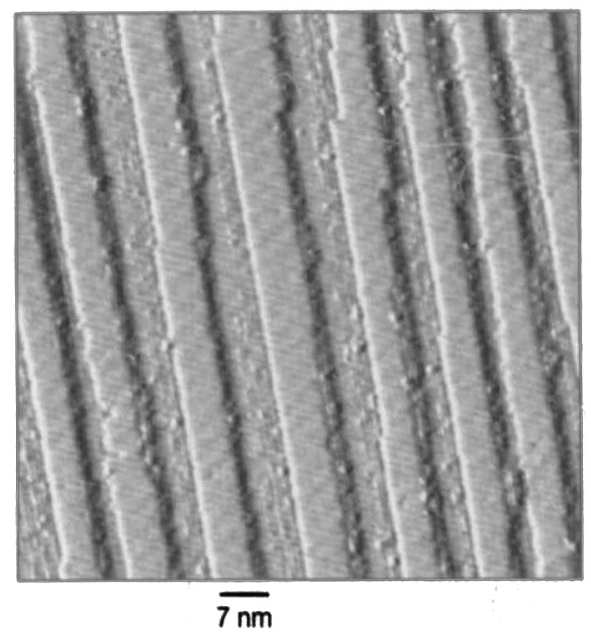

Figure 7. Epitaxial $\mathrm{CaF}_{2}$ mask deposited on a stepped Si(111) $(7 \times 7)$ surface $\left(100 \times 100 \mathrm{~nm}^{2}\right) . \mathrm{CaF}_{2}$ stripes ( $7 \mathrm{~nm}$ wide) grow on top of a complete $\mathrm{CaF}$, layer. From Reference 20.

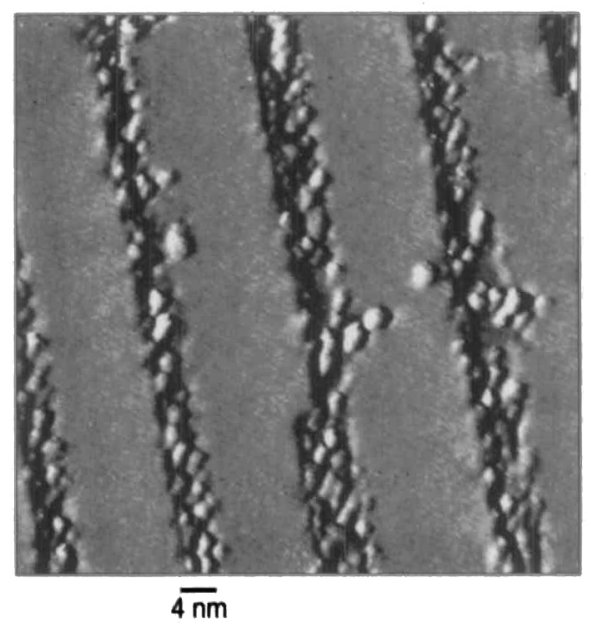

Figure 8. Selective deposition of organic molecules into $\mathrm{CaF}$, valleys between $\mathrm{CaF}_{2}$ stripes, using a structure similar to that in Figure 7 $\left(60 \times 60 \mathrm{~nm}^{2}\right)$. From Reference 22 


\section{Strings of Dots}

Steps might become useful templates not only for stripes, but also for strings of zero-dimensional objects. A first glimpse of such possibilities comes from an unusual growth mode of $\mathrm{CaF}_{2}$ on $\mathrm{Si}(111)$ at coverages below one-third of a monolayer (combined with temperatures below $700^{\circ} \mathrm{C}$ where $\mathrm{CaF}_{2}$ does not decompose into $\mathrm{CaF}_{1}$ ). As shown in Figure 9, the $\mathrm{CaF}_{2}$ stripes break up into strings of dots. They are attached to the bottom of the step edges, which is consistent with the normal stacking sequence observed for the F-terminated interface. The dots are about $10 \mathrm{~nm}$ in size, which is smaller than dot arrays produced by most other techniques $\left(80 \mathrm{~nm}\right.$ for $\mathrm{Cr}$ dots, ${ }^{23} 35-100 \mathrm{~nm}$ for SiGe dots, ${ }^{24} 70-210 \mathrm{~nm}$ for AlGaAs dots, ${ }^{25} 20 \mathrm{~nm}$ for holes etched into $\mathrm{Si}^{26}$ and 5-100 $\mathrm{nm}$ for holes etched into alumina ${ }^{5}$ ). A mechanism for creating homogeneous, self-assembled dots has also been proposed for SiGe on $\mathrm{Si}(100) .{ }^{24,27}$ The optimum dot size is determined by competition between the surface energy, which causes ripening into larger dots, and misfit strain, which makes large dots unstable. Whether an analogous process holds for $\mathrm{CaF}_{2}$ dots on $\mathrm{Si}(111)$ is currently being investigated. ${ }^{27}$

It is appealing to consider magnetic clusters or insulated $\mathrm{Si}$ dots lined up into one-dimensional strings along steps. These look like prototype memory cells for single-particle-per-bit data storage. Linear readout architectures could become feasible, such as a shift register or a

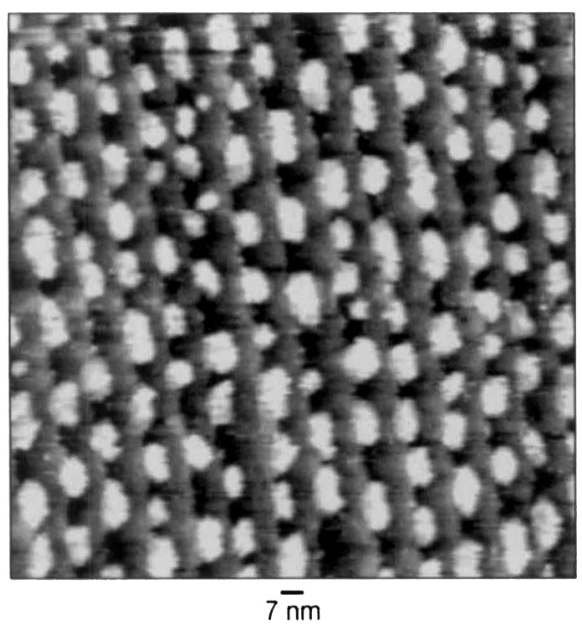

scanning probe moving parallel to the steps. The dots in Figure 9 have a density of $3 \times 10^{11} \mathrm{~cm}^{-2}$, or 2 teradots $/$ in. ${ }^{2}$, using units familiar to the storage industry. ${ }^{28}$ That exceeds commercially available bit densities in hard disks and opticalrecording media by almost three orders of magnitude. Of course, there are quite a few processing steps required for producing a viable storage array beyond establishing a mask, and it will be necessary to utilize extensions of techniques for etching ${ }^{5,26,29}$ and deposition ${ }^{22,30}$ in the nanometer regime. As an avenue for the future, the recent synthesis of nearly monodispersed, colloidal Co nanoparticles ${ }^{31}$ opens the possibility of assembling well-controlled arrays of magnetic dots attached to stepped silicon surfaces.

\section{Acknowledgments}

This work was supported by the U.S. National Science Foundation under award Nos. DMR-9632527 and DMR-9815416.

\section{References}

1. F.J. Himpsel, J.E. Ortega, G.J. Mankey, and R.F. Willis, Adv. Physs. 47 (1998) p. 511.

2. F.J. Himpsel, T. Jung, and P.F. Seidler, $I B M$ J. Res. Dev. 42 (1998) p. 33.

3. H. Röder, E. Hahn, H. Brune, J.-P. Bucher, and K. Kern, Nature 366 (1993) p. 141.

4. L. Piraux, J.M. George, J.F. Despres, C Leroy, E. Ferain, R. Legras, K. Ounadjela, and A. Fert, Appl. Phys. Lett. 65 (1994) p. 2484; A Blondel, J.P. Meier, B. Doudin, and J.-Ph. Ansermet, Appl. Phys. Lett. 65 (1994) p. 3019; K. Liu, K. Nagodawithana, P.C. Searson, and C.L. Chien, Phys. Rev. B 51 (1995) p. 7381

5. D. Routkevitch, A.A. Tager, J. Haruyama, D. Almawlawi, M. Moskovits, and J.M. Xu IEEE Trans. Electron Devices 43 (1996) p. 1646 Z.B. Zhang, J.Y. Ying, and M.S. Dresselhaus, J. Mater. Res. 13 (1998) p. 1745.

6. A.M. Morales and C.M. Lieber, Science 279 (1998) p. 208; G. Che, B.B. Lakshmi, C.R. Martin, E.R. Fisher, and R.S. Ruoff, Chem. Mater. 10 (1998) p. 260

7. F.J. Himpsel, Y.W. Mo, T. Jung, J.E. Ortega, G.J. Mankey, and R.F. Willis, Superlattices Microstruct. 15 (1994) p. 237.

8. T. Jung, R. Schlittler, J.K. Gimzewski, and F.J. Himpsel, Appl. Phys. A 61 (1995) p. 467. 9. P.M. Petroff, Ultramicroscopy 31 (1989) p. 67. 10. M. Paunov and E. Bauer, Appl. Phys. A 44 (1987) p. 201; M. Mundschau, E. Bauer, and W. Swiech, J. Appl. Plays. 65 (1989) p. 581.

11. Y.W. Mo and F.J. Himpsel, Phys. Rev. $B 50$ (1994) p. 7868

12. D.Y. Petrovykh, F.J. Himpsel, and T. Jung Surf. Sci. 407 (1998) p. 189; D.Y. Petrovykh, F.J.
Himpsel, and T. Jung (private communication). 13. T. Jung, Y.W. Mo, and F.J. Himpsel, Plyys. Rev. Lett. 74 (1995) p. 1641.

14. J. de la Figuera, M.A. Huerta-Garnica, J.E Prieto, C. Ocal, and R. Miranda, Appl. P/lys Lett. 66 (1995) p. 1006; J. Shen, R. Skomski, M - Klaua, H. Jenniches, S. Sundar Manoharan, and J. Kirschner, Phys. Rev. B 56 (1997) p. 2340. 15. T. Jung, F.J. Himpsel, R.R. Schlittler, and J.K. Gimzewski, in Scaming Probe Microscopy, Annlyticnl Methods, Chapter 2, edited by R. Wiesendanger (Springer, Berlin, 1998) p. 11. 16. J. Viernow, D.Y. Petrovykh, A. Kirakosian, J.-L. Lin, F.K. Men, M. Henzler, and F.J. Himpsel, Phys. Rev. B 59 (1999) p. 10356.

17. J. Viernow, J.-L. Lin, D.Y. Petrovykh, F.M Leibsle, F.K. Men, and F.J. Himpsel, $A p p l$. Phys. Lett. 72 (1998) p. 948.

18. J.-L. Lin, D.Y. Petrovykh, J. Viernow, F.K. Men, D.J. Seo, and F.J. Himpsel, I. Appl. Plyys 84 (1998) p. 255.

19. E.D. Williams and N.C. Bartelt, Science 251 (1991) p. 393.

20. J. Viernow, D.Y. Petrovykh, F.K. Men, A Kirakosian, J.-L. Lin, and F.J. Himpsel, Appl. Phys. Lett. 74 (1999) p. 2125.

21. D.Y. Petrovykh, J. Viernow, J.-L. Lin, F.M. Leibsle, F.K. Men, A. Kirakosian, and F.J. Himpsel, J. Vac. Sci. Techmol., A 17 (1999) p. 1415. 22. H. Rauscher, T. A. Jung, J.-L. Lin, A. Kirakosian, and F.J. Himpsel, Chem. Phys. Lett. 303 (1999) p. 363; J.-L. Lin, H. Rauscher, A. Kirakosian, and F.J. Himpsel (unpublished) 23. R. Celotta, R. Gupta, R.E. Scholten, and J.J. McClelland, J. Appl. Phys. 79 (1996) p. 6079.

24. J. Tersoff, C. Teichert, and M.G. Lagally, Phys. Rev. Lett. 76 (1996) p. 1675; C. Teichert, M.G. Lagally, L.J. Peticolas, J.C. Bean, and J. Tersoff, Physs. Rev. B 53 (1996) p. 16334; T.I. Kamins and R.S. Williams, Appl. Plyss. Lett. 71 (1997) p. 1201.

25. R. Nötzel, J. Temmyo, and T. Tamamura, Nature 369 (1994) p. 131.

26. M. Park, C. Harrison, P.M. Chaikin, R.A. Register, and D.A. Adamson, Scicuce 276 (1997) p. 1401

27. A.H. Li, F. Liu, and M.G. Lagally, Bull. Am. Phys. Soc. 44 (1999) p. 1714.

28. For an overview of ultrahigh-density recording technologies, see M.H. Kryder, MRS Bull. 21 (9) (1996) p. 17.

29. J.J. Boland and J.H. Weaver, Phys. Todny 51 (8) (1998) p. 34; R. Younkin, K.K. Berggren, K.S. Johnson, M. Prentiss, D.C. Ralph, and G.M. Whitesides, Appl. Phys. Lett. 71 (1997) p. 1261.

30. T.-C. Shen, C. Wang, G.C. Abeln, J.R. Tucker, J.W. Lyding, Ph. Avouris, and R.E. Walkup, Science 268 (1995) p. 1590; D.P. Adams, T.M. Mayers, B.S. Swartzentruber, Appl. Phys. Lett. 68 (1996) p. 2210; H. Dai, N. Franklin, and J. Han, Appl. Phys. Lett. 73 (1998) p. 1508

31. Shouheng Sun and C.B. Murray, J. Appl. Plyys. 85 (1999) p. 4325

Figure 9. Array of $\mathrm{CaF}_{2}$ dots (about $7 \times 10 \mathrm{~nm}^{2}$ in size), lined up along steps of a $\operatorname{Si}(111)(7 \times 7)$ surface $\left(200 \times 200 \mathrm{~nm}^{2}\right)$. Their density is $3 \times 10^{11} \mathrm{~cm}^{-2}\left(2\right.$ teradots $\left./ \mathrm{in}^{2}\right)$. From Reference 20. www.mrs.org/membership/ 